\title{
Un nouvel Hildegardia de la Réunion (Orthoptera, Caelifera,
}

\section{Tetrigoidea)}

\section{Sylvain Hugel}

\section{Abstract}

A new Hildegardia from Reunion island (Orthoptera, Caelifera, Tetrigoidea). A new species of Hildegardia Günther, 1974, is described from Réunion island: $H$. reuniivaga n. sp. A key to the known Hildegardia species is given.

\section{Résumé}

Une nouvelle espèce du genre Hildegardia Günther, 1974, est décrite de la Réunion : H. reuniivaga n. sp. Une clé des espèces de Hildegardia est donnée.

Citer ce document / Cite this document :

Hugel Sylvain. Un nouvel Hildegardia de la Réunion (Orthoptera, Caelifera, Tetrigoidea). In: Bulletin de la Société entomologique de France, volume 112 (4), décembre 2007. pp. 427-432;

https://www.persee.fr/doc/bsef_0037-928x_2007_num_112_4_16461

Fichier pdf généré le 07/10/2019 


\title{
Un nouvel Hildegardia de la Réunion (Orthoptera, Caelifera, Tetrigoidea)
}

\author{
par Sylvain HugEL \\ UMR 7168 ULP/CNRS, 21 rue René-Descartes, F-67084 Strasbourg cedex <hugel@neurochem.u-strasbg.fr> \\ Résumé. - Une nouvelle espèce du genre Hildegardia Günther, 1974, est décrite de la Réunion: H. reuniivaga n. sp. \\ Une clé des espèces de Hildegardia est donnée. \\ Summary. - A new Hildegardia from Réunion island (Orthoptera, Caelifera, Tetrigoidea). A new species \\ of Hildegardia Günther, 1974, is described from Réunion island: H. reuniivaga n. sp. A key to the known \\ Hildegardia species is given.
}

Key words. - Mascarene, Indian Ocean, Réunion, Mauritius, endemism, pigmy grasshopper.

Le genre Hildegardia a été créé par Klaus GüNTHER (1974) pour inclure deux espèces endémiques de l'île Maurice: $H$. mauritiicola Günther, 1974, et $H$. mauritiivaga Günther, 1974. Ces deux espèces arboricoles et arbusticoles vivent actuellement à l'île Maurice dans les milieux les plus préservés à partir de $500 \mathrm{~m}$ d'altitude pour $H$. mauritiicola et plus haut pour $H$. mauritiivaga (obs. pers.). Jusqu'à ce jour, ce genre était considéré comme endémique de l'île Maurice. En 2002, à la Réunion, dans la Vallée Heureuse, j'ai aperçu dans mon filet fauchoir un spécimen de Tetrigoidea n'appartenant pas à l'espèce Paratettix scaber (Thunberg, 1815), seul Tetrigoidea alors connu de cette île. Depuis, au prix de nombreuses nuits de terrain, il a été possible de rassembler une petite série de spécimens de ce Tetrigoidea très discret, proche mais bien distinct de $H$. mauritiivaga. Dans le présent article, Hildegardia reuniivaga n. sp. est décrit, des éléments dc son ćcologic ct de sa biologie sont donnés. Les caractères discriminants des trois espèces du genre Hildegardia sont illustrés et une clé des espèces du genre est proposée.

\section{Hildegardia reuniivaga n. sp.}

HOLOTYPE : đ, océan Indien, Mascareignes, île de la Réunion, Saint-Philippe, Basse-Vallée, Vallée-Heureuse, rempart est, sentier du Puys-Ramond, $896 \mathrm{~m}$ alt., $21^{\circ} 20^{\prime} 14^{\prime \prime} \mathrm{S}-55^{\circ} 42^{\prime} 15^{\prime}$ 'E, 21.IV.2007, S. Hugel, MNHN. AllotYPE: $q$, idem. PARATYPES : + , idem, CIRAD Réunion; 9 , idem, 03.IV.2005, $N$. Cliquennois, coll. S. Hugel; $\delta$, île de la Réunion, Bras-Panon, plaine des Lianes, forêt de la cascade du Chien, $765 \mathrm{~m}$ alt., 21 ${ }^{\circ} 02^{\prime} 05^{\prime}$ 'S-55'36'23''E, 21.IV.2007, S. Hugel, MSIRI (Mauritius Sugar Industry Research Institute); $\partial$, idem, CIRAD Réunion; $\delta$, idem, coll. H. Devriese, IRSNB, Bruxelles. JUVÉNILE : idem holotype, 14.XII.2005, R. Hugel leg. et coll.

Diagnose. - Cette espèce nouvelle est proche de $H$. mauritiivaga, mais s'en distingue clairement par la taille plus grande de 15 à $20 \%$ pour la longueur et la largeur du pronotum et des fémurs postérieurs tant chez les mâles que les femelles ${ }^{1}$ (voir fig. 5-8 et tableaux I et II). $H$. reuniivaga $\mathrm{n}$. sp. se distingue aussi nettement de $H$. mauritiivaga par les caractères suivants ( $\delta$ et $\$$ ): le bord antérieur du pronotum (légèrement anguleux chez reuniivaga $\mathrm{n}$. sp., droit chez mauritiivaga, fig. 5-8); la carène médiane du pronotum en vue latérale s'abaissant progressivement chez reuniivaga $n$. sp. mais brusquement chez mauritiivaga fig. 5-8); les expansions des articles antennaires (courtes et émoussées chez reuniivaga n. sp., longues et spiniformes chez mauritiivaga fig. 11-13); la carène médiodorsale des fémurs antérieurs (à peine ondulée chez $H$. reuniivaga n. sp., avec deux lobes chez $H$. mauritiivaga fig. 15-16).

\footnotetext{
'Différences statistiquement significatives (t-test $; \mathrm{p}<0,05$ )
} 
Description. - (Outre les caractéristiques générales du genre selon GÜNTHER, 1974).

Couleur: tacheté de noir et brun, parfois de vert; pronotum avec souvent une tache transversale claire bordée de motifs noirs avant l'extrémité postérieure (fig. l et 9).

Tête. Vertex 1,2 fois plus large que la plus grande largeur de l'œil en vue dorsale, chez les $\hat{\delta}, 1,6$ fois chez les $q$; vertex à bord antérieur bilobé, carène longitudinale visible à la base (antérieurement), totalement absente postérieurement; écusson clypéal étroit, divisé à partir du milieu des yeux, aussi haut que les yeux (fig. 10, vue de face); yeux légèrement saillants, dépassant le vertex en vue dorsale; carènes transverses longues, saillantes, prolongées par une série de tubercules; occiput avec des tubercules de dimension variable. Antennes longues, implantées au niveau du bord inférieur des yeux; comprenant 13 articles, les articles 3-13 sont comprimés, arrondis ventralement, avec une carène dorsale; les carènes dorsales des articles 5 à 10 comprennent une courte expansion qui se prolonge à peine sur l'article suivant (fig. 13).

Pronotum (terminologie d'après DFVRIESE, 1991) caractéristique de la forme dite nanopronotale considérée comme néoténique; bord antérieur légèrement anguleux, convexe (fig. 1 et 9); carènes antérieures courtes, rectilignes, convergeant à peine postérieurement (vue dorsale; fig. 1, 7, 8 et 9); carènes inférieures très courtes, $<0,5$ fois aussi longues que les carènes antérieures (vue latérale); carène médiane s'élevant relativement régulièrement dès le bord antérieur et s'abaissant relativement régulièrement (avec quelques ondulations) jusqu'à l'extrémité distale du processus apical sans jamais remonter distinctement et sans passer sous le niveau des carènes ventrales (fig. 2, 7, et 8); carène dorsale très courte, placée sous le sommet de la carène médiane, à une hauteur inférieure à celle de la carène antérieure (vue latérale); carène humérale courte et irrégulière dirigée vers l'extrémité distale de la carène dorsale; carènes transverses bien nettes postérieurement, atteignant le sinus inférieur; aire scapulaire très large, polygonale, prolongée jusqu'à l'extrémité distale du processus apical, bord inférieur légèrement convexe après le sinus inférieur; carènes latérales non décelables; bord postérieur du pronotum beaucoup plus court que l'abdomen, dépassant à peine le milieu des fémurs postérieurs; pronotum couvert de tubercules de dimension variable, hormis dans deux zones quadrangulaires localisées au même niveau que la base des fémurs III (vue dorsale) et deux affaissements triangulaires distaux (vue dorsale); lobes du pronotum saillants en vue dorsale.

Abdomen. Tergites abdominaux recouverts de tubercules de taille variable; bord postérieur du tergite IX prolongé par un processus médian arrondi, orienté vers l'arrière, remontant légèrement postérieurement, comprenant des tubercules (fig. 17-18).

Pattes. Fémurs I avec une carène longitudinale dorsale élevée, légèrement ondulée; deux carènes longitudinales ventrales, la ventrale postérieure avec 3 expansions spiniformes; deux carènes longitudinales latérales sur la face postérieure (fig. 15); tibias I à section quadrangulaire, les quatre carènes serrulées; fémurs II avec une carène longitudinale dorsale élevée comprenant 2 lobes médians et un lobe spiniforme distal; une carène longitudinale ventrale antérieure élevée comprenant 3 lobes spiniformes et un lobe distal; deux carènes longitudinales latérales sur la face antérieure; tibias II à section quadrangulaire, les quatre carènes serrulées; carène dorsale postérieure élevée et ondulée; fémurs III avec une carène supérieure élevée, serrulée, avec 4-6 expansions spiniformes antégéniculaires de taille croissante vers l'extrémité distale, et une expansion spiniforme géniculaire; lobe géniculaire supérieur prolongé d'une expansion spiniforme sur la face interne et externe ; lobes géniculaires ventraux interne et externe prolongés postérieurement; carène ventrale avec 5-7 lobes (certains spiniformes); carène médiolatérale avec deux lobes spiniformes le proximal obtus, le second aigu; aires supéro-interne et supéro-externe avec une série de quelque 8 renflements transverses; tibias III à section quadrangulaire, les deux carènes dorsales avec des serrulations; avec 2 éperons apicaux de part et d'autre.

Mâle. - Tête: apex du vertex (carènes transversales) dépassant à peine les yeux (vue latérale). Pronotum : aire scapulaire à bord inférieur (après la convexité) légèrement concave jusqu'à l'extrémité distale du processus apical. Pattes: les extensions spiniformes antégéniculaires et géniculaires des fémurs I, II et III sont moins marquées chez.les $\delta$ que chez les $q$. Tibia I avec 7-8 éperons sur la carène ventrale antérieure (holotype: $7-8$ ) ; 2-5 éperons sur la carène ventrale postérieure (holotype $2-3$ ). Tibias II avec 4-6 éperons sur la carène ventrale antérieure (holotype: 4 ); 3 éperons sur la carène ventrale postérieure. Tibias III avec 8-12 épines dorsales sur les carènes externes et internes (holotype: 8-9). Epiphalle: pont avec deux callosités couvertes de tubercules; avec une protrusion dirigée vers l'avant comprenant des tubercules (fig. 21). Complexe phallique comme sur la figure 22.

Femelle. - Tête: apex du vertex (carènes transversales) dépassant nettement les yeux en hauteur (vue latérale, fig. 2). Pronotum : aire scapulaire à bord inférieur (après la convexité) subrectiligne jusqu'à l'extrémité distale du processus apical. Pattes: les extensions spiniformes anté-géniculaires et géniculaires 

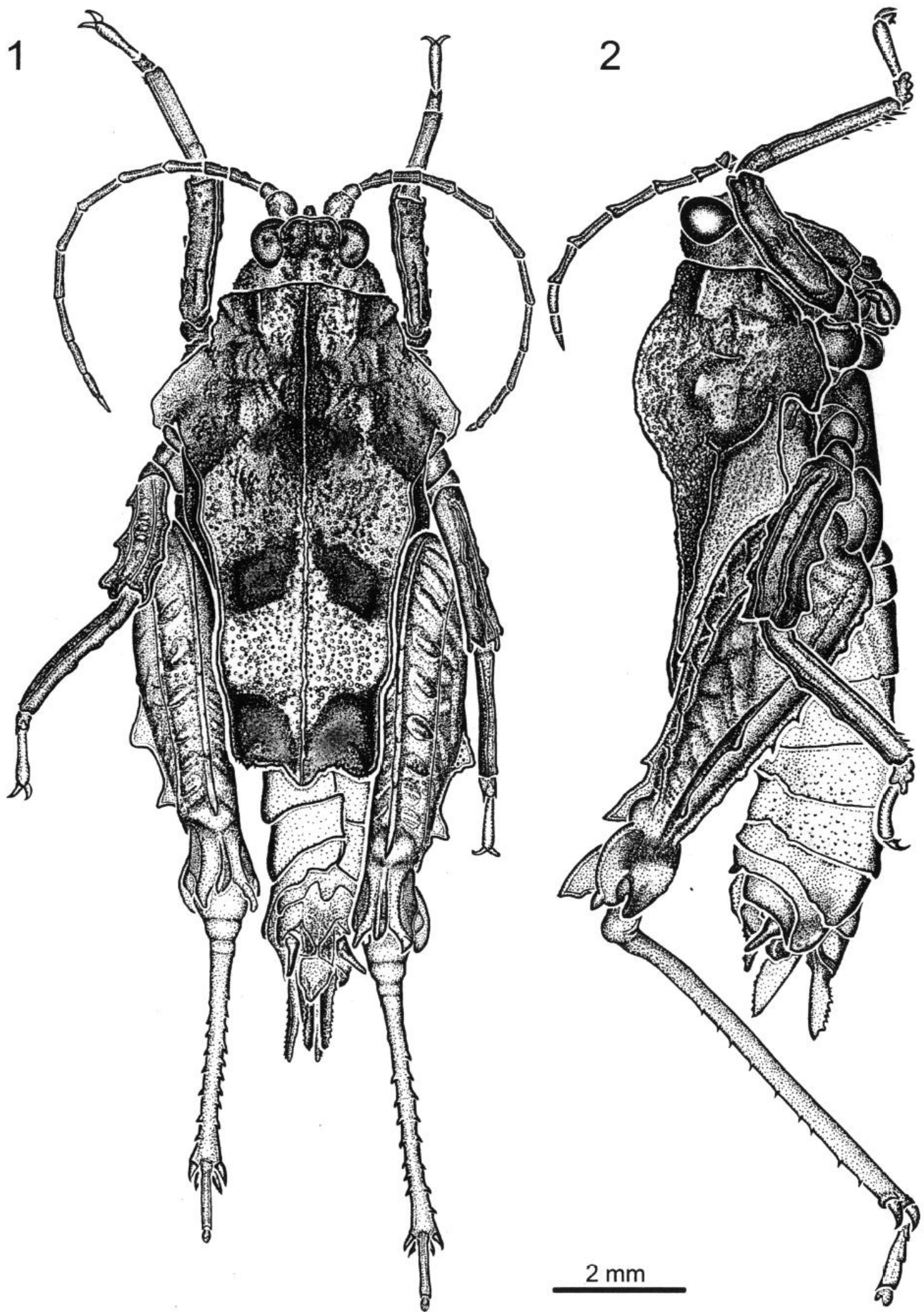

Fig. 1 et 2. - Hildegardia reuniivaga $n$. sp., $\subsetneq$ paratype.

les fémurs sont plus marquées chez les $q$ que chez les $\delta$. Tibias I avec 7-9 éperons sur la carène ventrale intérieure (allotype : 7) et 3 sur la postérieure; tibias II avec 3-5 éperons sur la carène ventrale antérieure allotype : 5) et 2-3 sur la postérieure (allotype: 3 ); tibias III avec 8 épines dorsales sur les carènes externes :t internes. Oviscapte : valves dorsales et ventrales avec chacune quelque 12 denticulations fines (fig. 14). 


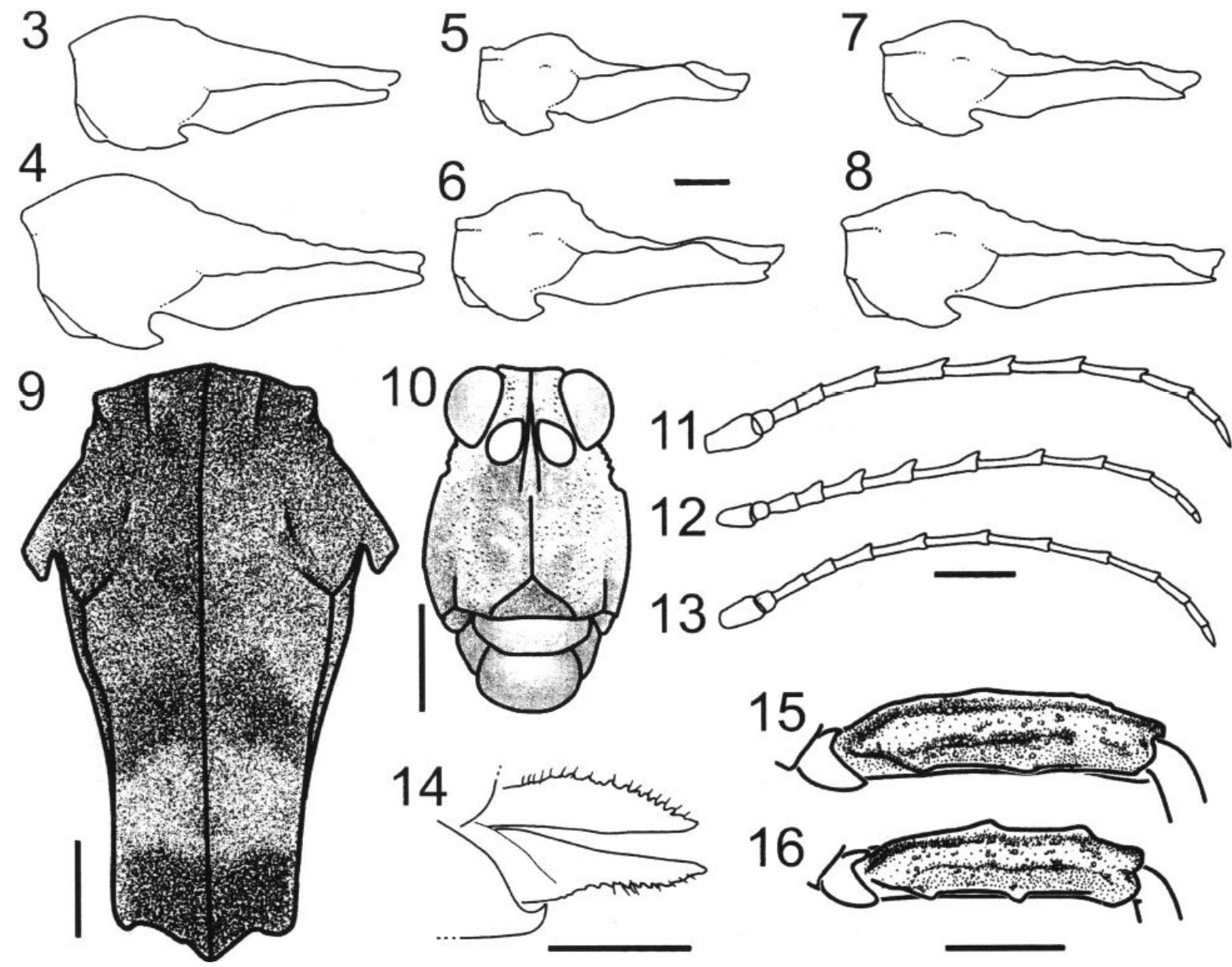

Fig. 3-16. - Hildegardia. - 3-8, Pronotum en vue latérale gauche, ô et $9 .-3-4$, H. mauritiicola Günther, 1974.5-6, H. mauritiivaga Günther, 1974. - 7-8, H. reuniivaga n. sp., holotype et allotype. $-9, H$. reuniivaga n. sp., $\delta$ holotype, pronotum en vue dorsale. - 10, Idem, face en vue frontale. - 11-13, Antenne droite, vue latérale : 11, H. mauritiicola, $q ; 12, H$. mauritiivaga, $q ; 13, H$. reuniivaga $\mathrm{n}$. sp., $q$ allotype. -14, H. reuniivaga $\mathrm{n}$. sp., $q$ allotype, oviscapte en vue latérale - 15-16, Tibia antérieur droit, vue postérieure (externe) ; 15, H. reuniivaga n. sp.. O allotype; 16, H. mauritiivaga Günther, 1974, \%. Barre d'échelle: $1 \mathrm{~mm}$.

Etymologie. - Le nom de cette espèce réunionnaise est construit comme celui de l'espèce mauricienne proche, décrite par Günther.

Tableau I. - Mesures de Hildegardia reuniivaga n. sp. (en mm).

\begin{tabular}{|c|c|c|c|c|c|c|c|}
\cline { 3 - 7 } \multicolumn{1}{c|}{} & \multirow{2}{*}{ Corps } & \multirow{2}{*}{ Antenne } & \multicolumn{3}{c|}{ Pronotum } & \multicolumn{2}{c|}{ Fémur postérieur* } \\
\cline { 4 - 8 } \multicolumn{1}{c|}{} & & & Longueur & Largeur & Hauteur & Longueur & Largeur \\
\hline \multirow{2}{*}{ h holotype } & 12 & 6,7 & 6,3 & 3,8 & 2,7 & 6,2 & 1,9 \\
paratypes $(\mathrm{n}=4)$ & $9,2-12,5$ & $6,2-6,9$ & $6,2-6,3$ & $3,8-4,1$ & $2,7-2,9$ & $6,2-6,5$ & $1,8-2,0$ \\
\hline $\begin{array}{c}\text { क allotype } \\
\text { paratypes }(\mathrm{n}=3)\end{array}$ & 13,3 & 7 & 7,6 & 4,8 & 3,2 & 6,6 & 2,1 \\
\hline
\end{tabular}

*Longueur du fémur postérieur jusqu'au lobe géniculaire dorsal (sans les expansions spiniformes).

Biologie. - H. reuniivaga n. sp. a été trouvé dans deux localités, à 750 et $900 \mathrm{~m}$ d'altitude, relativement éloignées l'une de l'autre $(25 \mathrm{~km})$. Les deux stations ont la même écologie, une pluviométrie supérieure à $7 \mathrm{~m}$ par an, une insolation inférieure à $1500 \mathrm{~h} / \mathrm{an}$, et sont constituées de fourrés hygrophiles à Pandanus bordés de forêt de Bois-de-Couleurs-des-Hauts. Dans les deux cas, il s'agit de milieux relativement préservés de la pression anthropique. Les neuf $H$. reuniivaga $\mathrm{n}$. sp. ont été observés en milieu de nuit: deux ont été pris par fauchage de la végétation basse; le spécimen juvénile est tombé dans une grande nasse, en secouant la végétation avec une barre télescopique de $5 \mathrm{~m}$; les six autres ont été collectés à vue, souvent par groupe, sur la 

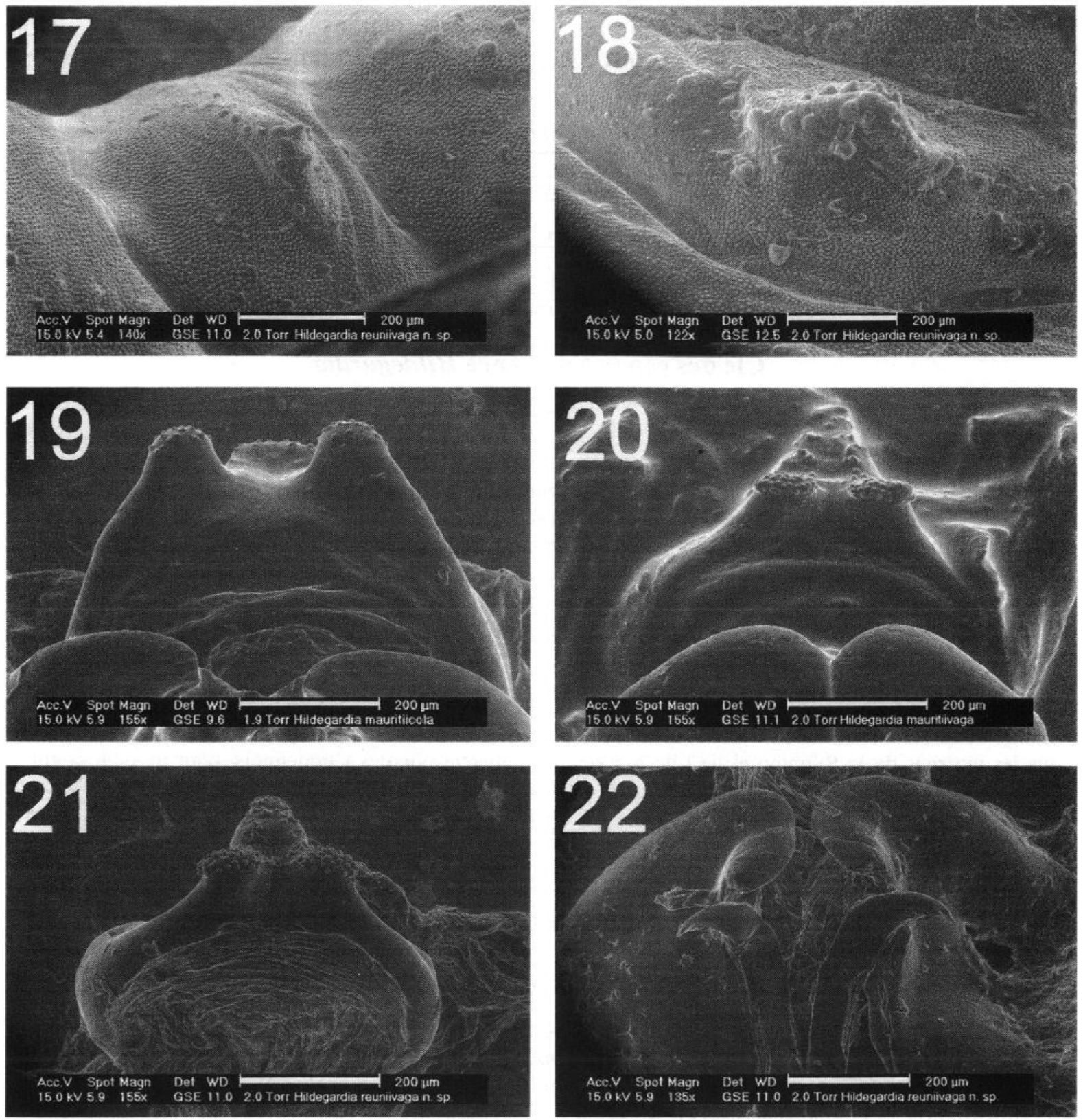

Fig. 17-22. - Hildegardia. - 17-18, H. reuniivaga n. sp., tergite $\mathrm{DX}$ en vue dorsale (avant : coin inférieur gauche), 17, $\delta$ holotype, 18, $q$ allotype. - 19-21. Epiphalle en vue dorsale; 19, H. mauritiicola Günther, 1974; 20, H. mauritiivaga Günther, $1974 ; 21, H$. reuniivaga $n$. sp., paratype. - 22, H. reuniivaga, paratype, complexe phallique en vue postérieure. Images réalisées, en mode environnemental, sans métallisation, avec le microscope électronique à balayage de l'Institut Pluridisciplinaire Hubert Curien, DEPE, UMR 7168, Strasbourg). Ech. : $200 \mu \mathrm{m}$.

mousse et les lichens recouvrant les branches à environ $2 \mathrm{~m}$ de haut. Aucun spécimen n'a pu être observé de jour. La capture d'un subadulte à la fin du mois de décembre et de tous les adultes durant le mois d'avril suggère un possible cycle saisonnier avec la présence d'adultes durant l'été austral.

Conclusion. - Hildegardia reuniivaga n. sp. est la troisième espèce de Caelifère endémique décrite de la Réunion (avec Gastrimargus immaculatus (Chopard, 1957) et Pyrgacris descampsi Kevan, 1976). Avec Paratettix scaber scaber (Thunberg, 1815) et les Acridiens Aiolopus thalassinus rodericensis (Butler, 1876), Gastrimargus africanus madagascariensis (Sjostedt, 1928), Locusta migratoria migratorioides (Reiche \& Fairmaire, 1850), Nomadacris septemfasciata (Audinet-Serville, 1838) et Anacridium melanorhodon arabafrum (Dirsh \& 
Uvarov, 1953), neuf espèces de Caelifères sont donc actuellement recensées à la Réunion (LUCAS, 1862 ; BOLIVAR, 1895 ; CHOPARD, 1957 ; TÊTEFORT \& WINTREBERT, 1965 ; DESCAMPS, 1968 ; KeVAN, 1975 ; HUGEL, 2005).

Tableau II. - Mesures de Hildegardia mauritiivaga Günther, 1974 (en mm).

\begin{tabular}{|c|c|c|c|c|c|c|c|}
\cline { 3 - 7 } \multicolumn{1}{c|}{} & \multirow{2}{c|}{ Corps } & \multirow{2}{*}{ Antenne } & \multicolumn{3}{c|}{ Pronotum } & \multicolumn{2}{c|}{ Fémur postérieur } \\
\cline { 4 - 8 } & & & Longueur & Largeur & Hauteur & Longueur & Largeur \\
\hline o allotype* & 7,8 & $?$ & 5,2 & 3,3 & 2,2 & $?$ & $?$ \\
min-max (n=2) & $7,8-9,0$ & 6,0 & $5,2-5,6$ & $3,3-3,4$ & $2,2-2,5$ & 5,7 & 1,6 \\
\hline o holotype* & 11,1 & $?$ & 6,5 & 4,0 & 2,9 & 6,2 & 2,0 \\
min-max (n=3) & $10,0-11,1$ & 6,6 & $6,5-6,6$ & $4,0-4,2$ & $2,9-3,1$ & $5,8-6,2$ & $1,9-2,0$ \\
\hline
\end{tabular}

*d'après Günther, 1974; les autres spécimens sont de Maurice, montagne Cocotte, 19-22.IV.2005, S. Hugel rec. et coll.

\section{Clé des espèces du genre Hildegardia}

1. Antennes de 12 articles (fig. 11); carènes antérieures du pronotum absentes, rarement à peine marquées (fig. 3-4). Epiphalle comme sur la fig. 19. Ile Maurice

H. mauritiicola Günther, 1975

- Antennes de 13 articles(fig. 12-13); carènes antérieures du pronotum courtes mais nettement marquées (fig. 5-8). Epiphalle comme sur les figures 20-21. Ile Maurice et île de la Réunion .............. 2

2. Carène dorsale des fémurs antérieurs avec au moins deux lobes/épines (fig. 16); épine distale des articles médians des antennes allongée (fig. 12), aiguë; pronotum comme sur les figures 5-6. Ile Maurice H. mauritiivaga Günther, 1975

- Carène dorsale des fémurs antérieurs ondulée en vue latérale, sans épine ou lobe distinct (fig. 15); épine distale des articles médians des antennes courte, émoussée (fig. 13); pronotum comme sur les figures 7-8. Ile de la Réunion.

H. reuniivaga n. sp.

RemerCIEMENTS. - Je remercie Alain Brondeau et l'Office national des Forêts ; Marylène Hoarau et le Parc national de la Réunion; Saoud Motala et la Mauritian Wildlife Foundation, pour m'avoir autorisé à collecter dans les réserves de la Réunion et de l'île Maurice. Je remercie Nicolas Cliquennois, pour m'avoir confié le spécimen qu'il a collecté. Merci à mon père, à mes collègues et amis entomologistes de la Réunion, particulièrement Antoine Franck du CIRAD et Jacques Rochat de l'Insectarium de la Réunion, qui m'ont accompagné lors de certaines collectes. Je remercie Jean-Hervé Lignot de l'Institut Pluridisciplinaire Hubert-Curien, DEPE, UMR 7168, Strasbourg, pour la réalisation des images au MEB. Enfin, je remercie la Société entomologique de France pour la Bourse Germaine Cousin (2001), le CIRAD Réunion qui a financé la mission de 2005, et le programme BIOTAS, ANR-06BDIV-002, grâce auxquels je peux mener mes investigations sur les Orthoptères des Mascareignes.

\section{AUTEURS CITÉS}

Bolivar I., 1895. - Mission scientifique de M. Ch. Alluaud aux îles Séchelles (mars-avril-mai, 1892), $6^{\mathrm{e}}$ mémoire : Orthoptères. Annales de la Société entomologique de France, 64 : 369-386.

ChOPARD L., 1957. - La faune entomologique de la Réunion. Orthoptérö̈des. Mémoires de l'Institut Scientifique de Madagascar, série E, 8: 31-56.

DESCAMPS M., 1968. - Un Acridoïde relique des Mascareignes (Orth. Acridoidea). Bulletin de la Société entomologique de France, 73 : 31-36.

DEVRIESE H., 1991. - Contribution à l'étude des Tetrigidae de Madagascar (Orthoptera). Bulletin et Annales de la Société Royale Belge d'Entomologie, 127 : 119-131.

GÜNTHER K., 1974. - Beitrag zur Kenntnis der Tetrigoidea (Orthoptera, Caelifera) von Madagascar und von Mauritius. Bulletin du Muséum national d'Histoire naturelle [Paris] ( $3^{e}$ sér.) Zoologie, (3) 236: 937-1031.

HUGEL S.. 2005. - Redécouverte du genre Pyrgacris à l'île de la Réunion: description du mâle de $P$. descampsi Kevan, 1975 (Orthoptera, Caelifera). Bulletin de la Société entomologique de France, 110 (2): 153-159.

KEVAN D.K.MCE., 1976. - Book Reviews. Grasshoppers. South African Journal of Science, 72: 316-319.

LuCAS P.H., 1862. - Orthoptères. In: Maillard L., Notes sur l'ile de la Réunion, t. 2, ann. i, 22-25, pl. 21 ; Paris.

TÊTEFORT J.P. \& WINTREBERT D., 1965. - Notes de mission au sujet de Nomadacris septemfasciata (Serville) et de Locusta mirgratoria L., à Maurice et à la Réunion. Agronomie Tropicale (Nogent), 20: 649-656.

UVAROV B. P., 1928. - The Orthoptera (excluding Blattidae) of Rodriguez Island. Annals and Magazine of Natural History, (10) 2: 362-364. 